\title{
Información, comunicación y cultura en la gestión local: la experiencia municipal en un territorio de vulnerabilidad de la ciudad de Montevideo
}

\author{
Paulina Szafran *
}

Artículo recibido:

29 de julio de 2014.

Artículo aceptado:

24 de febrero de 2015.
Resumen

El artículo describe una experiencia desarrollada en un territorio de la ciudad de Montevideo, Uruguay, caracterizado por su vulnerabilidad social. A partir de la reciente conformación en el país de los municipios como un tercer nivel de gobierno, indaga cómo funcionan en la gestión local del Municipio F la información, comunicación y cultura y de qué modo son concebidas por los actores municipales. Aborda en este contexto la percepción sobre la biblioteca pública en tanto servicio local. La metodología se sustentó en entrevistas a personas vinculadas a la gestión municipal de la zona de referencia y en el análisis de la documentación generada y difundida por dicho órgano.

* Universidad de la República (UdelaR-Uruguay), Montevideo, Uruguay. paulina.szafran@fic.edu.uy

INVESTIGACIÓN BIBLIOTECOLÓGICA, Vol.30, Núm. 70, septiembre/diciembre, 2016, México, ISSN: 0187-358X. pp. 19-39 
Palabras clave: Gestión Municipal; Políticas Locales; Bibliotecas Públicas; Información, Comunicación y Cultura.

\begin{abstract}
Information, communication and culture under local management: the municipality experience in a socially vulnerable neighborhood of Montevideo Paulina Szafran

The paper describes an experience in a neighborhood known for its social vulnerability in the city of Montevideo, Uruguay, The recent establishment of municipalities as a third level of government prompted this research to examine how information, communication and culture operate under the local management of Municipality $\mathrm{F}$ and how these activities are viewed by local actors. Within this scenario, the role of the public library is described, stressing the specifics of services delivered locally. The research methodology entailed interviews with persons connected to municipal management of the reference area and analysis of documentation produced and disseminated by that body.
\end{abstract}

Keywords: Municipal Management; Local Policies; Public Libraries; Information, Communication and Culture

\title{
LA INVESTIGACIÓN UNIVERSITARIA EN BiblioteCOLOGÍA ORIENTADA A LA INCLUSIÓN SOCIAL
}

D esde los variados campos de acción en que el investigador en bibliotecología puede abordar sus áreas de estudio cobra especial relevancia aquella mirada que permita incidir en la realidad cotidiana, acompañando procesos históricos que contribuyen al desarrollo disciplinar con una visión social a la vez que a la mejora en la calidad de vida de los ciudadanos.

No obstante que "la literatura latinoamericana en bibliotecología, publicada a lo largo del siglo XX, muestra notoriamente que la relación entre 'bibliotecas públicas y comunidades vulnerables' no fue uno de sus primordiales objetos de investigación teórica y empírica, a pesar de que estos grupos 
sociales estuvieron presentes a la vista prácticamente de toda la sociedad", es posible contar con valiosos antecedentes desde el ámbito universitario, vinculado a la formación en bibliotecología en Uruguay, de estudio y acción en el medio, incluso desarrollando experiencias en servicios de información que no se han promovido por los naturales agentes responsables, como los gobiernos locales y nacionales.

Esta concepción se ve enriquecida a partir del proceso que permite la reciente creación de la Facultad de Información y Comunicación (FIC) de la Universidad de la República (UdelaR), nuevo centro universitario en el que convergen las ciencias de la información y la comunicación, y en donde se conforma el Grupo de Investigación en Información y Comunicación para la Inclusión y la Integración Social, GIISUR. ${ }^{2}$ Este grupo se orienta a consolidar una línea multi e interdisciplinaria que, tomando como eje que la información y comunicación se destine a sectores vulnerables, destaca la investigación con sustento en una realidad que requiere ser transformada, concebida como insumo para el impulso de políticas de información dirigidas a las ciudadanas y ciudadanos. Asimismo, busca la retroalimentación del cuerpo teórico de los dominios involucrados, generando conocimiento interdisciplinario en teoría de la información y la comunicación.

Considerando el antecedente desarrollado en el territorio del Zonal 9 de la ciudad de Montevideo 3 por parte del Departamento de Información y Sociedad de la ex Eubca (Escuela Universitaria de Bibliotecología y Ciencias Afines), actual Instituto de Información, encauzado a las adolescentes y jóvenes de contexto vulnerable, GIISUR llevó a cabo un estudio durante el lapso 2010-2012, en la misma zona, referido al proceso de información-comunicación de las políticas de salud pública centradas en adolescentes.

El abordaje se enmarcó en las recientes políticas públicas que se están implementando en Uruguay, especialmente el Sistema Nacional Integrado de Salud y las emanadas de la AGESIC (Agencia de Gobierno Electrónico y Sociedad de la Información) y Agenda Digital. El entorno presente del país es propicio para que los universitarios promuevan la generación de conocimiento, sustentado en el medio, induciendo una retroalimentación con los responsables del dictado y ejecución de las políticas. Desde esta mirada, se

1 Felipe Meneses Tello, "Bibliotecas y sociedad: el paradigma social de la biblioteca pública", 167.

2 Grupo responsable de la investigación "Las estrategias de información y la comunicación centradas en los jóvenes y adolescentes en el ámbito de la salud: análisis y propuestas (RAP-ASSE, Zona 9 de Montevideo)" ejecutada entre los años 2010-2012, coordinada por los profesores Martha Sabelli, Jorge Rasner y Raúl Ruggia; referente internacional profesor Juan José Calva González.

3 Martha Sabelli y Verónica Rodríguez Lopater (comp.), La información y las jóvenes en contextos desfavorables: construyendo puentes para la inclusión social desde la investigación. 
hace imperioso multiplicar los esfuerzos de investigación en la disciplina que fomenten la incidencia profesional en la apropiación de las posibilidades que las nuevas políticas ofrecen a los habitantes.

A partir de esta configuración se presenta un aporte contextualizado dentro del amplio y variado campo de la relación información-ciudadanía, el cual parte de que el ciudadano "que es también decisor, su toma de decisiones las ejerce frente a múltiples problemas que debe enfrentar en su vida laboral, comunitaria y familia"4 y que, siguiendo el planteo de la autora, se concibe como un miembro de grupos, en este caso ubicado en ámbitos locales y no como un individuo aislado, por lo tanto, integrante de una comunidad de práctica en el acceso y uso de información. Desde la bibliotecología, el abordaje de los recintos comunitarios ha tenido un trascendental valor en la construcción de sus objetos de estudio.

Actualmente, la incorporación del concepto community engagement (CE) al desarrollo y evaluación de programas habilita aportar una perspectiva dirigida a la función del profesional bibliotecólogo en la exclusión social, promoción de la democracia y contribución al capital social/humano/cultural. ${ }^{5}$ Con ello se genera una línea de investigación orientada al análisis de la biblioteca y su papel respecto al CE, atendiendo a la difusión de información comunitaria así como a la utilización de su espacio para la toma de decisiones.

Durante el 2013 se desplegó una experiencia por parte de GIISUR que permitió ampliar el espectro de actores con los que había trabajado en el territorio de la Zona 9 de la ciudad de Montevideo, actual Municipio F; jóvenes y adolescentes en situación vulnerable y mediadores de servicios locales, incluidos los bibliotecólogos. ${ }^{6}$ Ante la posibilidad de profundizar la indagación en el territorio de referencia, se optó por añadir la visión de los políticos y técnicos vinculados a la gestión municipal.

Hablar de ciudadanos y de información en el contexto local conlleva necesariamente la correspondencia a la organización que de manera histórica se enfoca a estos referentes, la biblioteca pública. A los fines del artículo, se reafirma la dimensión política de la biblioteca pública ${ }^{7}$ así como su papel de-

4 Martha Sabelli, La información y el ciudadano en el entorno de la Sociedad de la Información: percepción de los actores políticos y sociales en el Uruguay, 58.

5 Hui-Yun Sung, Mark Hepworth y Gillian Ragsdell, "Investigating essential elements of community engagement in public libraries: an exploratory qualitative study".

6 Martha Sabelli y Jorge Rasner, Informe final. Las estrategias de información y comunicación centradas en Los jóvenes y adolescentes en el ámbito de la salud: análisis y propuestas (RAP-ASSE, Zona 9 de Montevideo); Martha Sabelli, "Investigaciones sobre el comportamiento informativo de adolescentes y jóvenes en situación vulnerable: hacia una información inclusiva en Uruguay".

7 Orlanda Jaramillo, "La biblioteca pública, un lugar para la formación ciudadana: referentes metodológicos para el proceso de investigación”. 
cisivo en la formación ciudadana ${ }^{8}$ atendiendo al rol de la información y conocimiento como instrumento del ejercicio ciudadano, ${ }^{9}$ a la vez de conceptualizar a la información como un objeto de investigación facilitador de una perspectiva integral e interdisciplinaria. ${ }^{10}$ Asimismo, requiere contextualizar a los actores participantes como integrantes de procesos de transferencia de información. Recordemos que

por naturaleza, el ser humano es un usuario de la información, ya que él mismo la genera y la consume, y como usuarios de la información pertenecen a diferentes sectores sociales, por lo cual se puede llegar a una tipología de los mismos, según el sector social al que pertenece. ${ }^{11}$

En este sentido interesa citar a Jaramillo, pues

plantear la institucionalidad de la biblioteca pública desde la dimensión ciudadana implica adoptar una definición que supere la simple concepción de facilitadora del acceso a la información y al conocimiento, y que además plantee y dinamice acciones y vínculos que hagan posible la formación del sujeto -usuario- con capacidad para exigir y ejercer sus derechos, pero también para cumplir con los deberes que de éstos se desprenden, en la pretensión de formar al ciudadano, que no sólo es consumidor de información sino también productor, transmisor y comunicador de información y de conocimiento. ${ }^{12}$

Este aspecto cobra trascendencia al investigar el contexto uruguayo en tanto cuenta con una nueva situación en materia de descentralización promovida por la Ley 18.567 "Descentralización política y participación ciudadana" de febrero de 2010, creadora de los municipios como un tercer nivel de gobierno, otorgando énfasis a la escena local, plataforma de relevancia para el análisis de los servicios bibliotecarios al ciudadano.

\section{La descentralización y lo "local" como escenario de acción de las bibliotecas públicas}

La instauración de los municipios en Uruguay acompaña una tendencia que se ha instalado en otros países, especialmente en América Latina, de menor

8 Orlanda Jaramillo, Biblioteca pública, ciudadanía y educación social.

9 Orlanda Jaramillo, "La formación ciudadana, dinamizadora de procesos de transformación social desde la biblioteca pública”.

10 Sabelli, "Investigaciones...".

11 Juan José Calva González, "Información y adolescencia: búsqueda de información por los adolescentes", 73.

12 Jaramillo, Biblioteca pública..., 127. 
dependencia de la política local respecto a la nacional. La institucionalidad establece la existencia de municipios con autoridades electas por la ciudadanía y la instrumentación de acciones que conducen el desarrollo local, por ejemplo, los presupuestos participativos.

Desde la llegada del partido de izquierda, Frente Amplio, al gobierno del departamento de Montevideo en el año 1990, comienza a implementarse un proceso de descentralización que dividió a la capital de Uruguay en 18 zonas, cada una contaba con el Centro Comunal Zona (CCZ), correspondiente a los asuntos administrativos; la Junta Local que conformaba la instancia política y el Concejo Vecinal que reunía a vecinos y organizaciones sociales. Dicha ordenación no significaba la constitución de un tercer nivel de gobierno, hecho que se concreta a partir de la mencionada ley mediante la creación de los municipios. En éstos se destaca la presencia de una figura desconocida en el país, el alcalde, y el Concejo Municipal, que constituyen gobiernos locales electos por la ciudadanía.

Hablar de un municipio nos posiciona en un nuevo entorno de descentralización, entendiéndola (y diferenciándola con la desconcentración) como "la transferencia de funciones, atribuciones, responsabilidades y recursos desde un órgano central del Estado a un órgano menor"; ${ }^{13}$ de acuerdo a Veneziano se trata de "un instrumento para derivar hacia la periferia las demandas que se ejercen sobre el centro del aparato estatal". ${ }^{14}$ Esta autora también presenta los distintos enfoques contradictorios que conlleva, desde la vinculación a las "fuerzas vivas del mercado" (con un papel reductor del Estado) a los "sectores populares" así como la necesaria articulación entre "polos falsamente dicotómicos": Estado/Sociedad; global/local; público/privado.

El contexto propuesto para la investigación, aunque referido a un ámbito territorial concreto, permite introducir reflexiones sobre el rol que le compete a la biblioteca pública, comprobando al igual que en otras realidades

la muy insuficiente integración de la biblioteca pública en las políticas públicas de carácter estatal y gubernamental; políticas que articulen efectivamente los planes, programas, proyectos y demás acciones encaminadas a desarrollar la biblioteca pública y a insertarla en la vida política de las personas. ${ }^{15}$

13 Antonio Cardarello, "Muchas dudas, algunas certezas y escaso entusiasmo. Las elecciones municipales 2010 en Uruguay", 65.

14 Alicia Veneziano Esperón, Sustentabilidad política, reforma de Estado y participación ciudadana en la Intendencia Municipal de Montevideo, 2.

15 Orlanda Jaramillo, Didier Álvarez Zapata y Daniel Moncada P., "Políticas públicas para bibliotecas públicas: una propuesta de soluciones locales a problemas globales”, 16. 
Dentro de los variados tipos de descentralización ${ }^{16}$ interesa caracterizar la territorial (que envuelve la descentralización funcional y política), que encierra una transferencia de responsabilidades de los primeros niveles de gestión, la asignación de recursos y el involucramiento de la sociedad civil. Se trata del espacio más propicio para la participación ciudadana, no sólo desde el punto de vista electoral sino a través de la participación política individual y grupal en el sentido de contribución al desarrollo de las políticas públicas. Siguiendo este planteo, Veneziano aporta una conceptualización de municipio "como una red donde se vinculan: el Estado, en su expresión político-territorial; el gobierno, en una instancia periférica; y los actores políticos y socio-económicos de la sociedad local". ${ }^{17}$ Esta idea de los municipios como redes es compartida por otros autores y facilita la comprensión de la diversidad de protagonistas y vínculos que actúan en lo local, aspecto que particularmente importa indagar con referencia a la biblioteca pública a manera de servicio social y cultural. En este marco, cobra especial interés la mirada que se le otorga a la biblioteca pública, el "ethos esencial", "la utilidad social de la biblioteca pública", 18 vinculado con tres relaciones sustanciales: biblioteca-comunidad; biblioteca-pueblo; biblioteca-sociedad. De igual modo, la ubicación de la biblioteca como espacio de socialización política ${ }^{19}$ permite comprender el rol que se le confiere con relación a la identidad política, instrumento de presencia ciudadana en la esfera pública.

En tanto práctica reciente (se debe considerar que aún no ha finalizado el primer periodo de gestión de los municipios, que comenzaron a funcionar en julio de 2010), en el caso de Montevideo, aún las bibliotecas públicas dependen administrativamente de la Intendencia de Montevideo, gobierno departamental (segundo nivel de gobierno). Esta realidad no impide que, como servicios locales, necesiten trabajar conjuntamente con los municipios, encontrando variadas experiencias de relacionamiento en las diversas zonas de la ciudad.

\section{Estrategia METODOLÓGICA}

El marco descrito orienta las preguntas de investigación aplicadas al territorio del Municipio F de Montevideo. Tomando como eje el énfasis en materia

16 Arocena citado por Cardarello, "Muchas dudas..."

17 Veneziano Esperón, Sustentabilidad política..., 2.

18 Meneses Tello, "Bibliotecas y sociedad...", 164.

19 Yicel Nayrobis Giraldo Giraldo, Gloria Elena Román Bentancur y Ruth Elena Quiroz Posada, "La biblioteca pública como ambiente educativo para el encuentro ciudadano: un estudio de la Comuna I de Medellín”. 
de información y comunicación se buscó averiguar respecto a si los contextos locales favorecen la articulación de políticas en distintas áreas y cómo se conjugan las políticas locales con las departamentales y nacionales, así como si es posible identificar políticas bibliotecarias en el territorio.

La metodología aplicada recoge la referencia de las modalidades desarrolladas por GIISUR en el proyecto original. En este punto la indagación se sustentó en entrevistas a sujetos considerados relevantes en la gestión del Municipio F y en el análisis de la documentación e información difundida desde ese organismo.

Considerando los recintos de competencia de la labor municipal y atendiendo a los fines del estudio, las entrevistas se dirigieron a figuras ligadas al tema social, cultural y de comunicación. Asimismo, se otorgó especial acento a la mirada política que envuelve el trabajo local, siendo la entrevista al alcalde un insumo apreciable para el análisis.

Con base en las áreas mencionadas, se realizaron siete entrevistas: dos destinadas a actores políticos, una al referente de cultura, tres al social y la restante a una persona vinculada a la comunicación. Se adoptó una pauta que abarcó preguntas abiertas, posibilitando un diálogo extenso. A pesar de utilizarse idéntica guía para la totalidad de los entrevistados, al momento de su aplicación se adaptó al perfil de las personas acorde a sus cargos. Las 34 interrogantes planteadas se organizaron en seis bloques: identificación del entrevistado; datos referidos a su vínculo laboral; la caracterización del Municipio F; la descripción del entrevistado de su función y accionar del Municipio F; acciones universitarias desarrolladas en la zona, y apreciación del entrevistado sobre la gestión local, cubriendo en este punto la indagatoria acerca de la percepción respecto a políticas bibliotecarias, de información y culturales. Especialmente se ahondó en aquellas respuestas que apuntaban a temas de información y comunicación, tanto a la interna del Municipio como en la relación con la ciudadanía.

El análisis documental se dirigió a los medios de difusión municipal, con énfasis en el boletín de noticias en tanto modalidad de comunicación que recoge toda la información vinculada al municipio y cuya frecuencia es semanal, a partir de la suscripción se recibe vía correo electrónico. Posee titulares de noticias referidas al Municipio F que se amplían mediante enlaces a su página web. A su vez, incluye un ítem con datos de otros municipios. La suscripción al boletín de noticias permitió vislumbrar la cantidad de actividades promocionadas desde el órgano local. Durante el periodo septiembre 2013 -febrero 2014 se recibieron y analizaron 26 boletines de noticias. 


\section{El COMPlejo ENTRAMAdo DE LA GESTión LOCAL}

La experiencia profundizó el conocimiento de una zona caracterizada como extensa, diversa y que enfrenta múltiples carencias. Se constata la fuerte presencia de una población infantilizada, con altos índices de pobreza y falta de servicios.

Sobre esta identificación macro es posible entender las perspectivas presentadas a partir de los distintos sectores de la gestión municipal y cómo, más allá de los marcos de referencia, cada región en concreto reclama sus particularidades y necesidades de fortalecimiento de estrategias para el acercamiento a los vecinos.

Ante la descripción anterior resulta significativo conocer los criterios de selección de los tópicos que se abordarán. El Plan Municipal de Desarrollo ${ }^{20}$ expresa los propósitos, objetivos, líneas estratégicas y acciones a desarrollar. Refiere a cuatro líneas de acción dirigidas a los grandes temas: infraestructura, medio ambiente, integración social, producción económica. Siguiendo las especificidades de este tipo de organismos, este documento muestra la multiplicidad de ejes de actuación, cubriendo distintos ítems del territorio. En su planificación operativa anual para el año 2011 las primeras propuestas se perfilan a la materia de vialidad; se remarca para los fines de la investigación la contemplación de las operaciones a cargo del responsable de Comunicación: "Elaborar estrategias de socialización de la información en el Municipio"; "Generar y fortalecer canales de comunicación"; "Crear e implementar un área de comunicación a nivel municipal”. Aunque se plantea como una labor "Promover el desarrollo de emprendimientos culturales y centros ciudadano", en la descripción de las actividades que la concretan se citan múltiples servicios pero no hay mención a las bibliotecas. Cabe aclarar que en la presentación de los participantes en el Plan Municipal de Desarrollo se nombra un importante número de organizaciones de diversas características vinculadas al territorio, sin aparecer las bibliotecas, de donde se infiere que su ausencia en la elaboración del plan motiva la nula contemplación de esta institución.

Es indudable que vivimos un tiempo en el que desde varias áreas se promueve lo local, aumentando las competencias y responsabilidades hacia los gobiernos locales, muchas veces sin proporcionar los instrumentos que les permitan cumplir con sus funciones y, por lo tanto, limitando sus posibilidades a pesar de las expectativas que generan. De acuerdo a De Barbieri, ${ }^{21}$

20 Municipio F, Plan Municipal de Desarrollo.

21 María de Barbieri, Ioanna Grotiuz, Alejandra Marzuca y Graciela Viera, Estrategias de descentralización en Uruguay: sobre los gobiernos locales y la importancia de la dimensión política, de gestión y socio-politica para la gobernanza territorial. 
lo local supone un territorio con sentido y significaciones compartidas para sus habitantes por lo que conviven múltiples racionalidades y envuelve un esfuerzo permanente de negociación de distintas lógicas de acción. Esto determina que los participantes del gobierno local puedan ocupar un rol que trascienda la función de meros gestores y a la vez que la ciudadanía tenga una actitud que vaya más allá de la lógica de la demanda.

Este panorama se refleja en la práctica cotidiana en el territorio. Los integrantes del municipio de referencia afirman que ha sido un gran avance el pasaje a la figura del municipio ya que anteriormente eran meros administradores de lo enviado por el nivel de gobierno superior, y en esta nueva situación ellos directamente deciden y cuentan con más presupuesto, aunque no es suficiente. Asimismo, este reciente escenario provoca tensiones, por lo rápido de la implementación del flamante sistema así como por la falta de preparación de sus principales actores políticos en la puesta en marcha de herramientas de gestión. Uno de los entrevistados señala la dificultad de no haber podido lograr el "gobierno de cercanía" en tanto se presume que este modelo implica estar cercanos a la gente; es decir, a pesar de reconocer un mayor contacto con la población, no resulta suficiente.

Atendiendo a un tema que afecta en forma directa a la gestión de las bibliotecas públicas, tanto respecto a su dependencia administrativa como a su potencial actuación de servicio de información local, se indagó sobre la articulación de las políticas nacionales, departamentales y locales en igual territorio. En este punto hay acuerdo acerca de la necesidad de mayor integración, hecho ilustrado a través de numerosos ejemplos de programas promovidos desde la órbita departamental que consideran que no corresponden a lo que los vecinos de la zona precisan; al decir de uno de los actores, "esa tensión entre la descentralización y la centralización es muy fuerte”. Otra afirmación da cuenta de las fragmentaciones que un idéntico espacio puede provocar: "tenés tres o cuatro programas que están haciendo lo mismo, totalmente descoordinado, es una repetición de cosas y un gasto de recursos". Por otra parte, al tratarse de una experiencia reciente, se comprueba que el relacionamiento del poder Ejecutivo es mediante las intendencias pero aún no de los municipios; se presenta entonces un aspecto remarcado en los temas indagados: la incidencia de las características personales de los involucrados, en esta oportunidad con referencia a los vínculos del alcalde con el intendente.

Para el caso del Municipio F, el equipo asume como eje para la labor interna la complementariedad y articulación de las áreas de actuación, ya que en la actividad concreta en el territorio es constante la coexistencia de distintos programas sin la adecuada coordinación. 
Adentrándonos en la propia dinámica de la práctica en el territorio tomamos la descripción realizada por el sector cultural. El gestor cultural trabaja con las comisiones de vecinos y con los centros culturales en donde funciona un formato de gestión mixta que no está sistematizado ya que hay modelos disímiles. Su tarea tiene que ver con el fortalecimiento de esos lugares en conjunto con la comisión de vecinos, lo cual demanda un considerable trabajo. "El éxito de los esfuerzos depende más de la comisión de vecinos, de la voluntad, de la participación, de la militancia de los vecinos y vecinas que de la gestión del Estado. Los que ejecutan son ellos, los que abren, cierran, limpian, etcétera". A su vez describe otras complejidades del contacto con los ciudadanos: "Después te juntas con vecinos, dicen lo que sienten el resto de los vecinos y te dicen, acá a los vecinos la cultura no les importa”. La representatividad es un tópico complejo, a veces hay opiniones que no serían del colectivo pero que también son aportadas por quienes tienen los liderazgos de la comisión.

\section{LA EXPERIENCIA CON RELACIÓN A LAS BIBLIOTECAS PÚBLICAS}

A pesar de la vastedad del territorio analizado, son escasos los servicios bibliotecarios disponibles y su poca presencia quedó de manifiesto al no incluirse en el plan municipal descrito. En la zona es posible ubicar tres bibliotecas públicas, dos pertenecientes a la órbita departamental de la Intendencia de Montevideo (la Biblioteca Pública Francisco Espínola y la Biblioteca Pública Francisco Schinca, que además comparte pertenencia con otro municipio) y una dependiente del Ministerio de Educación y Cultura (Biblioteca José Batlle y Ordoñez). Se conoce la existencia de bibliotecas populares aunque, como es común en Uruguay, por las características que asumen no se puede determinar el número exacto de las que funcionan en la circunscripción.

Al vincular el tema municipal con las políticas de información, se indaga con los entrevistados respecto a las bibliotecas públicas y su rol como centros locales de información y la situación en la zona. Los actores del Municipio F visualizan a las bibliotecas y plantean distintas modalidades de funcionamiento remarcando como dificultad constante los horarios reducidos que estos servicios tienen. En un barrio concreto, los entrevistados del área social comentan que han discutido con los vecinos qué papel debería tener la biblioteca ya que para éstos era significante que la biblioteca participara: "Es un espacio referente o debe serlo". En este caso preciso la biblioteca pública de la zona había desarrollado varios años atrás una experiencia de trabajo 
con la comunidad, sobre todo de gestión con las organizaciones de vecinos, a instancia del profesional a cargo de la biblioteca, experiencia que aún permanecía en el recuerdo de sus integrantes.

Los entrevistados destacan especialmente a la Biblioteca Francisco Schinca ya que desde que está la nueva encargada ${ }^{22}$ ha pasado a tener una actuación de referente para el barrio, incorporando otros elementos como lo recreativo y lo educativo. Nuevamente se afirma que la actitud de la persona que esté al frente es fundamental para una buena labor, a lo que se agrega el compromiso profesional. Haciendo referencia a la importancia otorgada en América Latina a lo local, Revesz reclama en este contexto una actitud acorde del bibliotecario: "Más que en otra época, mucho de lo posible y de los resultados esperados depende de la capacidad del bibliotecario de no ser sólo un funcionario, un técnico entre cuatro paredes y un gestor de los recursos que ya tiene, sino además un abogado y un embajador de su institución hacia afuera". ${ }^{23}$

El trabajador vinculado a la gestión cultural del territorio entiende que es factible reconocer las políticas bibliotecarias a partir no sólo de la búsqueda de materiales o de la renovación de la biblioteca, y opina que desde el libro ya no se puede enfocar su misión: "hay que buscar métodos nuevos [...] hay que generar actividades como está haciendo ahora la Schinca, que tengan que ver con el libro, que tengan que ver con la lectura pero que no es ir a buscar un libro, eso es lo que va a hacer que la biblioteca funcione, que tiene un potencial enorme". El entrevistado describe un panorama más crítico con relación a las bibliotecas populares dada la baja presencia de usuarios y la necesidad de modificar las formas de administración: "tiene que ver con la gestión y con el hecho de dejar librado algo tan importante como es una biblioteca pública a la gestión vecinal".

A partir de la visión de uno de los actores políticos la falta de recursos se plantea como una limitante para el desarrollo de las bibliotecas de la zona, lo que repercute en una característica del territorio, la falta de espacios. Relata una anécdota que permite dar cuenta de esta afirmación: un niño de uno de los barrios del zonal lanzó una convocatoria a través de Facebook para organizar una biblioteca para su barrio. De esa forma recogió una importante cantidad de libros pero su madre llamó al municipio porque no podía tener ese volumen en su casa, desde el órgano local los levantaron y lo único que pudieron hacer fue llevarlos a otra casa. Ante la falta de locales se hicieron las gestiones para contar con un bibliobús y lo consiguieron, pero no se contrató

22 Desde diciembre de 2012 la biblioteca está a cargo de un profesional incorporado mediante llamado público de la Intendencia de Montevideo a Licenciados en Bibliotecología.

23 Bruno Revesz, "Políticas de información, bibliotecas públicas y desarrollo local y regional", 58. 
a una persona encargada ni se consideró la gestión. En concreto, está el bibliobús y una colección pero no hay personal por no disponer de presupuesto.

Respecto al tema del acceso a la información por la ciudadanía, el propio alcalde manifiesta que quizás haya condiciones para favorecerlo que no han sabido desarrollar. En el transcurso de la entrevista comenta que, posiblemente y a partir del diálogo que genera esa instancia, incorporar computadoras en el municipio para que los vecinos accedan sería un avance no difícil de definir. El hecho permite presumir que el reconocimiento del valor otorgado a la información para el ciudadano no se entiende a priori vinculado a la biblioteca pública pero, por otra parte, se encuentra una postura abierta a poder concretar acciones que no impliquen una importante movilización de recursos, escenario en el que se hace imperioso contar con la iniciativa del profesional bibliotecólogo. Reafirmando la línea de pensamiento de Jaramillo, ${ }^{24}$ resulta indudable que la participación como práctica ciudadana demanda del ciudadano ciertas condiciones pero también de la biblioteca.

Desde la perspectiva analizada interesa posicionar el desafío que significa para la biblioteca pública esta nueva condición institucional, destacando su rol con relación a la educación política, presentada por Ríos Ortega: "La elección de representantes, y en general participar en la vida pública construida alrededor del Estado y un sistema de gobierno, implica apropiarse de conocimiento e información pública. Es por tanto esencial subrayar que la ciudadanía también requiere educación política". ${ }^{25}$ Sin embargo, cuesta vislumbrar la admisión de esta mirada a la realidad en tanto que, a pesar de las potencialidades de las bibliotecas públicas, ya se había constatado la limitada incidencia de los servicios bibliotecarios para el ciudadano de la Zona $9^{26}$ y particularmente para el público adolescente y joven. ${ }^{27}$

\section{LA COMUNICACIÓN APLICADA A LA GESTIÓN MUNICIPAL}

El eje comunicación es resaltado en forma exhaustiva con los entrevistados, entendiendo que se trata de un aspecto transversal a los distintos ámbitos de trabajo. La competencia del sector es vasta, tendiendo a un mayor énfasis en el uso de las TIC. El área de comunicación genera los contenidos de la página web institucional, se encarga de las redes sociales (Facebook, Twitter y You-

24 Jaramillo, "La formación ciudadana...".

25 Jaime Ríos Ortega, "El acceso abierto a la información y la formación ciudadana”, 142.

26 Paulina Szafran Maiche, "Recursos de información para la ciudadanía: documentos, servicios de información y comunicación”.

27 Jimena Núñez Ansuas, "Servicios de información en la Zona 9 y las políticas de información en salud destinadas al público joven". 
Tube), y elabora y envía el boletín semanal de noticias (vía correo electrónico por suscripción); asimismo, edita una revista con un promedio de cinco números al año (publicación gratuita distribuida por los propios integrantes, disponible además en línea). Otros puntos se centran en participar en la programación de una radio comunitaria de la zona y confeccionar las carteleras que se colocan para el público en el local del Municipio F y Centro Comunal Zonal. ${ }^{28}$ Desarrolla además una instancia conjunta con actores universitarios, la intervención en Bien de cerca en la radio del Instituto de Comunicación de la FIC, un programa de promoción de los municipios. No menos importante es la atención a las variadas solicitudes que reciben, en la medida de las posibilidades, muchas veces sin contar con los recursos necesarios para la resolución; un ejemplo común lo constituye el pedido de afiches de los vecinos para difundir distintas acciones.

La mayor parte de los instrumentos reseñados fueron analizados con base en los fines de la investigación, especialmente el boletín de noticias. Su exploración corresponde con lo revelado en las entrevistas realizadas. Se presentan noticias vinculadas a iniciativas propias del Municipio F en relación con la política local, las muestras más claras son las relativas a tránsito, vialidad, iluminación y poda. Por otro lado, se difunden distintas informaciones que proporcionan colectivos de la zona, varias refieren a actos culturales, lo que ubica a la cultura como una materia de mucha difusión.

Atendiendo a las áreas temáticas, algunas se identifican a temas definidos, por ejemplo, social, cultura; otras $-\mathrm{y}$ como es deseable que ocurra en la ejecución de las tareas-involucran varios temas, por lo que no es posible agruparlas en una categoría determinada. Un tipo que interesa particularmente, por el ámbito de aplicación, son las actividades llevadas a cabo en la Biblioteca Francisco Schinca, como el programa La Infancia es Capital.

En el análisis se comprobaron las afirmaciones realizadas en las entrevistas respecto a la cobertura de las actividades. En algunos casos sólo se informa de su realización mientras que otras se difunden, después de llevarlas a cabo, a través de entrevistas a participantes, comentarios, etcétera. Algunas noticias se anuncian una vez mientras otras se repiten por varias semanas. Este hecho obedece a la importancia del asunto, como la elección de los concejos vecinales e información sobre el presupuesto participativo que son aspectos que hacen al gobierno local. La época del año en que se difundan también influye, por ejemplo, en el periodo correspondiente al mes de enero

28 Cabe aclarar que, a lo largo del proyecto "Hacia la construcción de Servicios de Información a la Comunidad: estudio del acceso y uso de información por las mujeres de contextos desfavorables (Zona 9 de Montevideo)", las carteleras ya habían sido identificadas por parte de los mediadores de los servicios de la zona como un importante medio difusor de información para la ciudadanía. 
es posible observar la repetición de noticias, seguramente por las pocas actividades que se realizan en ese mes.

El periodo de la cobertura permite dar cuenta del tipo de información difundida; de este modo, durante el mes de diciembre se difunden muchos actos referidos a cierres y muestras de talleres que se realizan en la zona, en el mes de febrero se celebra el carnaval y por lo tanto se repiten los datos de los desfiles en los barrios del territorio.

Por tratarse de un punto que interesa especialmente se resalta que la Biblioteca Francisco Espínola no es mencionada a lo largo de los boletines analizados, a la Biblioteca José Batlle y Ordoñez se la cita una vez mientras que la Biblioteca Francisco Schinca (que comparte territorio con otro municipio) aparece reiteradamente, confirmando la diversidad de acciones que realiza esta institución, hecho puntualizado por los entrevistados de todas las esferas.

La comunicación no queda exenta de las dudas que se generan en los entrevistados sobre la labor municipal con relación al acercamiento al vecino, específicamente referidas a las formas empleadas y a los medios de difusión. En distintas secciones se cuestiona que la información se difunda mayormente por vías electrónicas, lo que determina que llegue a quienes tienen acceso pero no siempre a los lugares que quisieran, por lo que se trata de una modalidad en la que es necesario mejorar. Desde el área cultural se recalca su valor en la medida en que la comunicación de las actividades puede provocar el éxito o fracaso de las mismas. Aparte de los medios aludidos, para temas culturales se han utilizado también autoparlantes.

La información-comunicación se presenta en otro ejemplo referido a la propia gestión municipal, acentuando la necesidad de informar sobre el tercer nivel de gobierno ya que se enfrentan en lo cotidiano al desconocimiento de la población acerca de la nueva institucionalidad y no es totalmente asumida al interior del equipo de trabajo en tanto, como funcionarios, entienden que la van aprendiendo en el transcurrir de la gestión diaria.

\section{Conclusiones}

Una primera aproximación permite afirmar que el naciente contexto ofrecido a partir de la implementación de los municipios, como un tercer nivel de gobierno, es adecuado con las características del territorio y propicia un mayor acercamiento con la población de la zona, promoviendo su participación y otorgando más autonomía en la gestión local. Sin embargo, a pesar de que la mayoría de los entrevistados entiende que la situación ha mejorado, tienen la sensación de que se podría hacer más. Por un lado se opina que, como to- 
da experiencia inicial, hay elementos que se conocen sobre la marcha de los acontecimientos y que esperan perfeccionar para el próximo periodo de ejercicio; igualmente hay situaciones que preocupan a los referentes con relación a la posibilidad de concretar en los hechos lo que encarna la instrumentación de los municipios. En este sentido, contar con mayores recursos se manifiesta como una de las principales necesidades para corregir y ampliar muchas de las acciones. Este aspecto, mencionado anteriormente como relevante para la descentralización, no siempre se corresponde con lo que sucede en su puesta en práctica y con el énfasis que se le confiere en la actualidad a la gestión local.

Cabe remarcar el convencimiento de que no es sólo un tema económico, también se vincula con dos ítems que se plantean reiteradamente: el poco saber que existe aún respecto a lo que significan los gobiernos municipales y la convicción de que las características de las personas que se encuentran al frente de las iniciativas y organizaciones que se ubican en el territorio determinan muchas de las particularidades de los servicios y acciones que allí se llevan a cabo.

A pesar de las contrariedades destacadas con relación a la duplicación de programas y recursos, se entiende que el ámbito local puede actuar como articulador en lo atinente a la aplicación de distintas políticas, tanto atendiendo a las diversas temáticas involucradas como a los niveles de los órganos responsables. Los actores participantes confían en que el proceso de implementación de los municipios, sobre todo el conocimiento de lo que implican, posibilite regular estos aspectos y mejorar, aunque no se percibe como una tarea sencilla.

Tomando el ejemplo de las bibliotecas públicas de Montevideo es posible comprobar que la propia estructura institucional confluye en ese sentido, la instalación de los municipios no alteró su dependencia central de la Intendencia de Montevideo, por lo que el vínculo con los gobiernos locales (en algunos casos como la mencionada Biblioteca Schinca, que actúa en territorios correspondientes a más de un municipio) obedece a otras condicionantes, muchas veces limitadas a las buenas o malas relaciones de las personas.

A partir de la metodología desarrollada, no se detectaron elementos que den cuenta de la identificación de políticas bibliotecarias en el territorio. Sin embargo, interesa resaltar que los entrevistados reconocen la importancia de las bibliotecas y su carácter de servicios locales, otorgándoles un papel que trasciende el rol tradicional de estas unidades y concibiéndolas como espacios sociales y culturales beneficiosos en el territorio, si bien la persona que se encuentra al frente de las mismas es la que marca las características de la unidad más allá de las políticas que se diseñen. A su vez, constituye un punto en donde se ratifica la carencia de recursos, fundamentalmente de personal, lo que conlleva a la mayor crítica, el horario reducido y todas las consecuen- 
cias que esta circunstancia genera. Queda planteada la interrogante sobre si la concepción de la biblioteca como servicio local, presentada por muchos de los entrevistados, tiene que ver con el hecho de la reciente incorporación de una profesional en una de las bibliotecas y su inserción en la zona, en particular si se considera que el plan mencionado fue elaborado previamente a este cambio en la biblioteca. Esta situación comprueba la expresión de Meneses Tello: "lo que se hace a través del servicio de biblioteca pública y cómo se hace, en el entramado de los diversos grupos sociales, adquiere suma importancia". ${ }^{29}$

La información y comunicación son apreciadas como insumos trascendentes en el acercamiento a la población, instrumentos valiosos para lograr la efectiva participación que hace a la esencia de la gestión local y que se concibe difícil de concretar. Aunque dicho reconocimiento no está directamente unido a la biblioteca. La recalcada necesidad de difusión de la gestión local y la importancia de los municipios no se visualiza con la presencia de la biblioteca, empero, no hay una postura contraria a esto, por lo que una propuesta en esa dirección sería apreciada. Desde este marco sería posible profundizar el rol de aquélla, siguiendo a Ríos Ortega, no porque le corresponda a la biblioteca la generación de información derivada del ejercicio del gobierno pero sí con relación al acceso a la información pública, "así como la guía experta que el ciudadano requiere para su decodificación". ${ }^{30}$ La concepción planteada del ciudadano en tanto decisor, integrante de una comunidad, conlleva para su efectiva concreción de estos instrumentos y del espacio de la biblioteca pública como ámbito propicio de conjunción con el vecino;

Desde esta perspectiva, este tipo de bibliotecas deben continuar contribuyendo alrededor del mundo a desarrollar más y mejores capacidades y habilidades entre los miembros de la sociedad civil para el ejercicio de sus acciones ciudadanas a realizar en el interior de sus comunidades. ${ }^{31}$

De acuerdo con los objetivos propuestos para esta instancia, resulta destacable la extensión del diálogo con los actores de la zona. En los antecedentes de la investigación había sido remarcado el gran nivel de inserción logrado, hecho que pudo comprobarse en esta oportunidad. A pesar de la vinculación con nuevos interlocutores existía conocimiento del trabajo del grupo y las acciones realizadas en el territorio, reafirmando la convicción de que el diálogo conjunto, por el aporte de las distintas miradas que proporcionan los saberes de los actores participantes, permite intervenir en la realidad para transformarla.

29 Meneses Tello, "Bibliotecas y sociedad...", 168.

30 Ortega, "El acceso abierto...", 143.

31 Meneses Tello, "Bibliotecas y sociedad..." 170. 


\section{Bibliografía}

Calva González, Juan José. "Información y adolescencia: búsqueda de información por los adolescentes", en Memoria del Tercer Seminario Hispano-Mexicano de Investigación en Bibliotecología y Documentación. Tendencias de la Investigación en Bibliotecología y Documentación, Filiberto Felipe Martínez Arrellano y Juan José Calva González (comp.), 73-89. México: IIBI, 2006. Fecha de consulta: 14 de julio de 2014, http://cuib.unam.mx/libros.html

Cardarello, Antonio. "Muchas dudas, algunas certezas y escaso entusiasmo. Las elecciones municipales 2010 en Uruguay". Revista Uruguaya de Ciencia Política 20 (1) (2011): 63-94. Fecha de consulta: 10 de junio de 2014, http://www.scielo.edu.uy/pdf/rucp/ v20n1/v20n1a04.pdf

Carrillo, E. "La nacionalización de la política local”. Política y Sociedad 3 (1989): 29-46. Fecha de consulta: 15 de junio de 2014, http:// revistas.ucm.es/index.php/POSO/article/download/POSO89892 30029A/30654

De Barbieri, María, Ioanna Grotiuz, Alejandra Marzuca y Graciela Viera. Estrategias de descentralización en Uruguay: sobre los gobiernos locales y la importancia de la dimensión política, de gestión y socio-política para la gobernanza territorial, Montevideo: Flacso Uruguay, 2010.

Giraldo Giraldo, Yicel Nayrobis, Gloria Elena Román Bentancur y Ruth Elena Quiroz Posada. "La biblioteca pública como ambiente educativo para el encuentro ciudadano: un estudio de la Comuna I de Medellín”. Revista Interamericana de Bibliotecología 32 (1) (2009): 47-84. Fecha de consulta: 17 de noviembre de 2014, http:// aprendeenlinea.udea.edu.co/revistas/index.php/RIB/article/ view/2749

Herzer, H., P. Pírez. "Municipio y participación popular en América Latina”. Desarrollo Económico 29 (114) (1989): 187-205. Fecha de consulta: 19 de junio de 2014, http://www.jstor.org/stable/34669 61

Jaramillo, Orlanda. Biblioteca pública, ciudadanía y educación social, Buenos Aires: Alfagrama, 2013.

- "La formación ciudadana, dinamizadora de procesos de transformación social desde la biblioteca pública”. Revista Interamericana de Bibliotecología 35 (1) (2012): 73-82. Fecha de consulta: 17 de noviembre de 2014, http://www.redalyc.org/articulo.oa?id= 179024991007

"La biblioteca pública, un lugar para la formación ciudadana: referentes metodológicos para el proceso de investigación”. Revista Interamericana de Bibliotecología 33 (2) (julio/diciembre 2010): 287-313. Fecha de consulta: 7 de julio de 2014, http://aprendeenli nea.udea.edu.co/revistas/index.php/RIB/article/view/7644/7069 
Jaramillo, Orlanda, Didier Álvarez Zapata y Daniel Moncada P. "Políticas públicas para bibliotecas públicas: una propuesta de soluciones locales a problemas globales". Investigación Bibliotecológica 19 (39) (julio/diciembre 2005): 13-27. Fecha de consulta: 7 de julio de 2014, http://www.scielo.org.mx/scielo.php?pid=S0187-358 X2005000200002\&script $=$ sci_arttext

Ley $N^{\circ} 18.567$. Descentralización política y participación ciudadana. Fecha de consulta: 25 de junio de 2014, http://www.parlamento.gub. uy/leyes/AccesoTextoLey.asp?Ley $=18567 \&$ Anchor $=$

Meneses Tello, Felipe. "Bibliotecas y sociedad: el paradigma social de la biblioteca pública”. Investigación Bibliotecológica 27 (61) (septiembre/diciembre 2013): 157-173. Fecha de consulta: 21 de junio de 2014, http://www.revistas.unam.mx/index.php/ibi/article/ download/42818/38893

Municipio F. Plan Municipal de Desarrollo, 2010. Fecha de consulta: 21 de junio de 2014, http://municipiof.montevideo.gub.uy/system/files/pmd.pdf

Núñez Ansuas, Jimena. "Servicios de información en la Zona 9 y las políticas de información en salud destinadas al público joven”, en Estrategias de información y comunicación en salud centradas en adolescentes, M. Sabelli y J. Rasner (comp.), Montevideo: PRODIC (en prensa), 2013.

Revesz, Bruno. "Políticas de información, bibliotecas públicas y desarrollo local y regional". Revista Interamericana de Bibliotecología 24 (2) (julio/diciembre 2001): 45-63. Fecha de consulta: 7 de julio de 2014, http://aprendeenlinea.udea.edu.co/revistas/index.php/ RIB/article/viewFile/3198/2963

Ríos Ortega, Jaime. "El acceso abierto a la información y la formación ciudadana”, en Regulaciones que impactan la infodiversidad y el acceso abierto a la información en la sociedad global y multicultural, Estela Morales Campos (comp.), 135-148. México: IIBI, 2013. Fecha de consulta: 21 de junio de 2014, http://132.248.242.6/ publi$\mathrm{ca} /$ conmutarl.php? arch $=4 \& \mathrm{idx}=1157$

Sabelli, Martha. "Investigaciones sobre el comportamiento informativo de adolescentes y jóvenes en situación vulnerable: hacia una información inclusiva en Uruguay", en Usuarios de la información en diferentes comunidades académicas y sociales: investigaciones, Juan José Calva González (comp.), 77-104. México: IIBI, 2013. Fecha de consulta: 4 de julio de 2014, http://132.248.242.6/ publi$\mathrm{ca} /$ conmutarl.php? arch $=4 \& \mathrm{idx}=1089$

_ y Jorge Rasner. Informe final. Las estrategias de información y comunicación centradas en los jóvenes y adolescentes en el ámbito de la salud: análisis y propuestas (RAP-ASSE, Zona 9 de Montevideo), Montevideo: Prodic, 2012.

— y Verónica Rodríguez Lopater (comp.). La información y las jóvenes en contextos desfavorables: construyendo puentes para la inclusión social desde la investigación, Montevideo: CSIC, 2012. 
Sabelli, Martha. La información y el ciudadano en el entorno de la Sociedad de la Información: percepción de los actores políticos y sociales en el Uruguay, Montevideo: Ediciones de la Banda Oriental, 2008.

Sung, Hui-Yun, Mark Hepworth y Gillian Ragsdell. "Investigating essential elements of community engagement in public libraries: an exploratory qualitative study", Journal of Librarianship and Information Science 45 (3) (2013): 206-218. Fecha de consulta: 19 de noviembre de 2014, http://lis.sagepub.com/content/45/3/206

Szafran Maiche, Paulina. "Recursos de información para la ciudadanía: documentos, servicios de información y comunicación", en La información y las jóvenes en contextos desfavorables: construyendo puentes para la inclusión social desde la investigación, Martha Sabelli y Verónica Rodríguez Lopater (comp.), 161-178. Montevideo: CSIC, 2012.

Veneziano Esperón, Alicia. La participación en el diseño e implementación de políticas públicas en la descentralización del gobierno municipal de Montevideo (1990-2000), trabajo presentado al V Congreso del CLAD, 2000. Fecha de consulta: 20 de junio de 2014, http:// siare.clad.org/fulltext/0039229.pdf

- Sustentabilidad política, reforma de Estado y participación ciudadana en la Intendencia Municipal de Montevideo, trabajo presentado en el V Congreso Nacional de Ciencia Política-Sociedad Argentina de Análisis Político, 2001. Fecha de consulta: 20 de junio de 2014, http://www.saap.org.ar/esp/docs-congresos/congresos-saap/V/docs/diaz/alicia-veneziano.pdf

"Izquierda y descentralización en Montevideo", Ecuador Debate 61 (2004). Fecha de consulta: 10 de junio de 2014, www.platafor mademocratica.org/Publicacoes/5303.pdf

Gobernanza en la descentralización: una mirada desde América Latina, trabajo presentado en VII Congreso Español de Ciencia Política y de la Administración: Democracia y Buen Gobierno, 2005. Fecha de consulta: 20 de junio de 2014. http://www.aecpa. es/uploads/files/congresos/congreso_07/area03/GT10/VE-

NEZIANO-Alicia.pdf

"La participación ciudadana en la descentralización de Montevideo: aprendizajes y reflexiones desde los noventa”, Revista Uruguaya de Ciencia Política 17 (1) (2008): 203-227. Fecha de consulta: 20 de junio de 2014, http://www.scielo.edu.uy/scielo.php?script =sci_pdf\&pid=S0797-97892008000100009\&lng =es \&nrm=iso \& tlng $=$ es

- Relaciones intergubernamentales, intragubernamentales y socio-gubernamentales en el decreto de descentralización de Montevideo, trabajo presentado en X Jornadas de Investigación de la Facultad de Ciencias Sociales, 2011. Fecha de consulta: 20 de junio de 2014, http://www.fcs.edu.uy/archivos/Veneziano.pdf 


\section{Para citar este artículo:}

Szafran, Paulina. 2016. "Información, comunicación y cultura en la gestión local: la experiencia municipal en un territorio de vulnerabilidad de la ciudad de Montevideo." Investigación Bibliotecológica: Archivonomía, Bibliotecología e Información 70: 19-39. http:// dx.doi.org/10.1016/j.ibbai.2016.10.002

$\infty$ 